\title{
Experimental Space-Division Multiplexed Polarization-Entanglement Distribution through 12 Paths of a Multicore Fiber
}

\author{
Evelyn A. Ortega $\odot,{ }^{1,2, *}$ Krishna Dovzhik, ${ }^{1,2}$ Jorge Fuenzalida, ${ }^{1,2, \S}$ Sören Wengerowsky $\odot,{ }^{1,2,}$, \\ Juan Carlos Alvarado-Zacarias, ${ }^{3}$ Rodrigo F. Shiozaki, ${ }^{4}$ Rodrigo Amezcua-Correa, ${ }^{3}$ \\ Martin Bohmann $\odot,{ }^{1,2, \dagger}$ and Rupert Ursin $\odot^{1,2, \$}$ \\ ${ }^{1}$ Institute for Quantum Optics and Quantum Information - IQOQI Vienna, Austrian Academy of Sciences, \\ Boltzmanngasse 3, Vienna 1090, Austria \\ ${ }^{2}$ Vienna Center for Quantum Science and Technology (VCQ), Vienna, Austria \\ ${ }^{3}$ CREOL, The University of Central Florida, Orlando, Florida 32816, USA \\ ${ }^{4}$ Departamento de Física, Universidade Federal de São Carlos, Rodovia Washington Luís, km 235-SP-310, \\ São Carlos, SP 13565-905, Brazil
}

(Received 18 August 2021; revised 14 October 2021; accepted 12 November 2021; published 21 December 2021)

\begin{abstract}
The development and wide application of quantum technologies highly depend on the capacity of the communication channels distributing entanglement. Space-division multiplexing (SDM) enhances data channel transmission capacity in classical telecommunication and bears the potential to transfer the idea to quantum communication using current infrastructure. Here, we demonstrate a SDM of polarizationentangled photons over a $411 \mathrm{~m}$ long 19-core multicore fiber distributing polarization-entangled photon pairs through up to 12 channels simultaneously. The quality of the multiplexed transfer is evidenced by high polarization visibility and Clauser-Horne-Shimony-Holt (CHSH) Bell inequality violation for each pair of opposite cores. Our distribution scheme shows high stability over $24 \mathrm{~h}$ without any active polarization stabilization and can be effortlessly adapted to a higher number of channels. This technique increases the quantum-channel capacity and allows the reliable implementation of quantum networks of multiple users based on a single entangled photon pair source.
\end{abstract}

DOI: 10.1103/PRXQuantum.2.040356

\section{INTRODUCTION}

Quantum communication has been rapidly progressing in private and secure information transmission [1-4]. In any quantum communication scheme, one needs communication channels to distribute the quantum information encoded in properties of photons between the users. The

\footnotetext{
*evelyn.ortega@oeaw.ac.at

†martin.bohmann@oeaw.ac.at

†rupert.ursin@oeaw.ac.at

$\S$ Current address: Fraunhofer Institute for Applied Optics and Precision Engineering IOF, Albert-Einstein-Str. 7, Jena 07745, Germany

"Current address: ICFO-Institut de Ciencies Fotoniques, The Barcelona Institute of Science and Technology, Barcelona, Castelldefels 08860, Spain
}

Published by the American Physical Society under the terms of the Creative Commons Attribution 4.0 International license. Further distribution of this work must maintain attribution to the author(s) and the published article's title, journal citation, and DOI. major types of channels are free-space links [5,6], satellite [7], and optical fibers [8-10], where fiber connections are the easiest and already deployed option for the last mile connection to the customers. In this context, a vital aspect of optimizing resources is the development and implementation of high-capacity quantum channels.

Today multiplexing is widely used in classical telecom infrastructure [11] transmitting data in parallel to increase the capacity of a single deployed channel. Space-division multiplexing $(\mathrm{SDM})$ is currently being investigated in the classical communications context [12]. This multiplexing approach relies on few-mode $[13,14]$, multimode [15], and multicore fibers [16-18], as well as novel mutiplexers and demultiplexers $[19,20]$ to harness the spatial dimension of light and boost capacity compared to single-mode fiber systems. In quantum communication schemes, multiplexing technologies have been implemented in different degrees of freedom, such as time [21], wavelength [22], and polarization [23]. However, the multiplexing of photons in terms of their spatial modes over optical fibers has not been thoroughly explored yet in an experiment. In terms of fibers links, single-mode fibers (SMFs) are not suitable for SDM implementations since only one 
spatial mode can be carried. In contrast, the fibers capable of transmitting multiple spatial modes, such as multiand few-mode fibers, require a complex system [24] to avoid the mode-coupling effects [25] and are susceptible to external influences [26] inhibiting long-term stability. A multicore fiber (MCF) is superior in this sense because all the spatial modes are guided through individual cores that share the same cladding [27]. The MCF preserves the distinguishability of discrete propagation paths while assuring the low crosstalk between the cores.

In the context of quantum information, MCF recently gained popularity [28], where, e.g., the parallel transmission of quantum and classical signals has been shown $[29,30]$. However, to improve quantum-communication applications, it is necessary to devise strategies that enable the parallel transmission of quantum information over the same channel. The multiple paths available in MCF have been a useful, they have been a useful tool for exploring high-dimensional quantum communication [31-34], transmission of four-dimensional spatially entangled photon pairs [35,36], and generation of a multidimensional entanglement state [37]. However, the huge potential of exploiting quantum correlations in different degrees of freedom for MCF communication has not been tackled so far.

Here, we present an experimental implementation where a MCF is used as a communication channel to simultaneously distribute multiple entangled photon pairs independently of each other from a single entangled photon pair source. We report on the characterization of the coupling and distribution of polarization-entangled photons through a 19-core fiber, demonstrating excellent performance of the system. In particular, we certify high path visibilities, i.e., the quality of correlations between opposite fiber cores, and that the polarization entanglement between two opposite cores is well preserved over the MCF as well as in the fiber-splice-based separation of the paths. We demonstrate long-term stability, relevant for applications, of the quantum SDM setup. To the best of our knowledge, this is the first experimental realization of a polarization-entanglement distribution scheme based on momentum correlation over a MCF and the simultaneous use of quantum correlations in multiple degrees of freedom. Our results show that quantum SDM can be achieved by exploring intrinsic momentum correlations in the spontaneous parametric down-conversion (SPDC) process, which is not possible via random distribution of the photons.

\section{IMPLEMENTATION}

The experimental setup is schematically depicted in Fig. 1(b). Photon pairs are generated by a type- 0 phasematched SPDC source in a Sagnac configuration (see Fig. 6 in Appendix A for more details). Through the

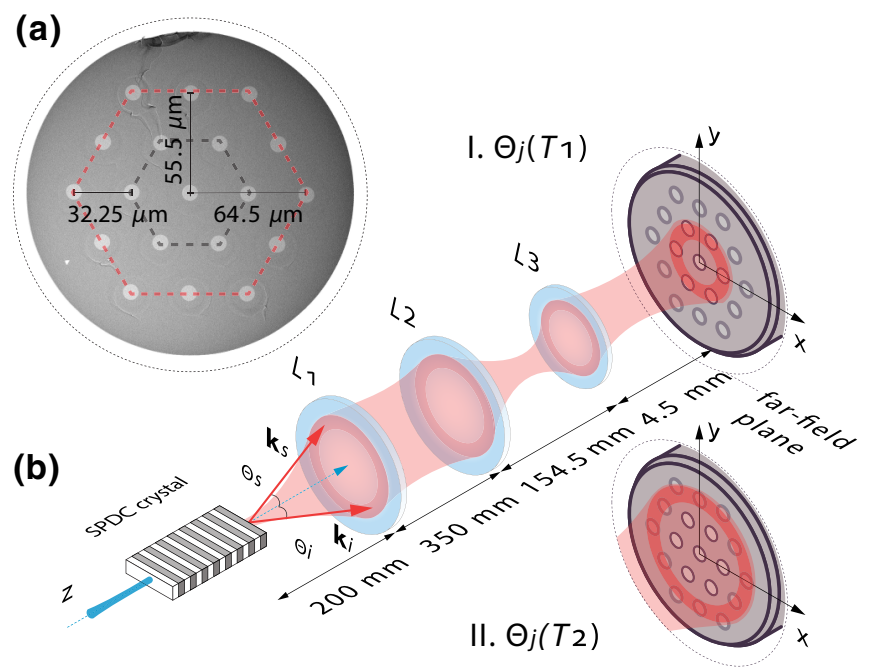

FIG. 1. Illustration of the implemented SDM through a 19core fiber. (a) An electron microscope image of the 19-core fiber. The inner and outer rings of cores are highlighted by the black and red hexagons, respectively. (b) A laser beam (blue, $z$ is the pumping direction) pumps a nonlinear crystal where the downconverted photons are created. The emitted photons form an emission cone (noncollinear type-0 phase matching) shown in red. Opening of the cone defined by the angles $\Theta_{s}$ and $\Theta_{i}$ for the signal and idler photons depends on the crystal temperature $T$. The lenses $L_{1}, L_{2}$, and $L_{3}$ image and demagnify the far-field plane of the crystal onto the MCF's end face. We show two cases for different cone openings $\Theta_{j}(T)$ for the temperature: (I) $T_{1}$ illuminating the inner ring of cores; (II) $T_{2}$ illuminating the outer ring of cores. Fiber end faces are not shown to scale.

nonlinear crystal operated in the Sagnac configuration, a polarization-entangled state is created. The momentum conservation in the SPDC process warrants that the signal and idler photons are emitted with opposite transverse components of the wave vectors $\left(\mathbf{k}_{s}\right.$ and $\left.\mathbf{k}_{i}\right)$ at the telecommunication wavelength. Hence, the photons of an entangled pair are located at diametrically opposite points in the far-field plane of the crystal, i.e., the transverse momentum representation of the photon pair [38,39]. By a cleverly devised lens system [see Fig. 1(b)], we image the far-field plane of the SPDC crystal to the end face of the MCF. Thus, opposite cores of the MCF are linked via the momentum correlations exhibited by the photon pairs. The resulting quantum state inside the MCF is a polarization-spatial hyperentangled state

$$
|\boldsymbol{\Phi}\rangle=\left|\Phi^{+}\right\rangle_{\text {pol }} \otimes \sum_{m=1}^{N} g_{m}\left|c_{m}, c_{m}^{\prime}\right\rangle_{\text {cores }}
$$

where the first part is the polarization-entangled Bell state and the latter part describes the spatial-entangled state as a high-dimensional superposition of opposite cores (paths), 
$c_{m}$ and $c_{m}^{\prime}$. Here, the $g_{m}$ are normalized probability amplitudes, $\sum_{m=1}^{N}\left|g_{m}\right|^{2}=1$, and $N$ is the number of opposite cores.

The lens $L_{1}$ performs a Fourier transform of the transverse position of the photons at the crystal plane [see Fig. 1(b)]; therefore, the far field is placed at the focal point of the lens $L_{1}$. The far-field plane is imaged and demagnified to match it to the end face of the MCF using a $2 f / 2 f^{\prime}$-imaging system consisting of the lenses $L_{2}$ and $L_{3}$ (see Appendix A for more details). The precise matching of the SPDC emission cone onto the rings of the MCF is achieved through temperature control of the nonlinear crystal. Here, we take advantage of the fact that the temperature influences the phase-matching condition of the SPDC process, resulting in a varying opening angle of the emission cone [40,41] [ $\mathbf{k}_{s}$ and $\mathbf{k}_{i}$ in the Fig. 1(b)].

The 19-core fiber was fabricated at CREOL in the University of Central Florida, where stack and draw methods were used in the fabrication process. A set of 19 trenchassisted core rods were placed in a hexagonal array inside a jacketing tube, then a set of packing rods of different sizes made out of silica were used to fill up the space between the core rods. Once the preform was assembled, the fiber was fabricated in our drawing tower. The fiber is designed to support the fundamental mode at $1550 \mathrm{~nm}$, its cores have a diameter of approximately $8.5 \mu \mathrm{m}$ with an index difference $\Delta n=5.5 \times 10^{-3}$ with respect to silica, whereas the trench has an index difference $\Delta n=-5 \times 10^{-3}$ with respect to silica. The 19 cores of the $411 \mathrm{~m}$ long MCF are arranged in an array with hexagonal symmetry with a coreto-core distance of approximately $32.25 \mu \mathrm{m}$ [see Fig. 1(a)]. The array of 19 cores naturally consists of a central core and two hexagonal rings of cores that we refer to as the inner ring (six cores closest to the central core) and the outer ring (12 cores at the circumference of the fiber end face) [see Fig. 1(a)]. A trench structure is implemented to reduce interchannel crosstalk. The MCF is connected to a fan-in-fan-out (FIFO) device that interfaced each core to a SMF with an insertion loss $\leq 3 \mathrm{~dB}$ per core. The FIFO is fabricated following the process described in Ref. [42]. Through a careful alignment of the MCF with respect to the emission cone of the SPDC source (see Appendix A) we realized a symmetrical intensity distribution at its end face and we can reliably change the phase matching to emit a cone to either illuminate the inner or outer ring by changing the temperature of the crystal.

To characterize the correlation between the distributed entangled photons in the spatial and polarization degrees of freedom, we use two detection configurations where the single channels of the MCF are either connected to photon detectors directly or pairwise through a pair of polarization analyzing modules, respectively (see Appendix A for details). Superconducting nanowire single-photon detectors (SNSPDs) with an efficiency of approximately $80 \%$ and dark-count rates of approximately $10^{2} \mathrm{~Hz}$ are used for the photon detection. The detection events are recorded with a time-tagging module for further analysis. Correlated photon pairs are identified through coincidences in counts with the help of temporal cross-correlation functions.

\section{PATH VISIBILITY}

We start with the characterization of spatial correlations of the photons distributed over the MCF. For this purpose, we measure the photon intensities and the coincidence counts between all possible combinations of cores in each ring (Fig. 6 in Appendix A, module a). All combinations of core pairs, 15 pairs for the inner ring and 66 pairs for the outer ring, are measured for $30 \mathrm{~s}$ each.

The SPDC source produces spatially entangled photons that show anticorrelations in momentum [43]. The transverse wave vectors of the signal and idler photons are mapped through the far-field measurement onto MCF cores (paths) [see also Fig. 1(b)]. In this way, we relate the momentum correlations to the path correlations between opposite cores of the rings.

The coincidence measurements between the different cores are shown in Fig. 2 (inner ring) and Fig. 3 (outer ring). Indeed, we observe the expected strong correlations between the opposite cores $m$ and $m^{\prime}$ while almost no coincidences are detected between any nonopposite cores. Although the MCF with the fan-out device shows different coupling efficiencies and transmission losses for each core, the behavior is equivalent for each core: high correlations
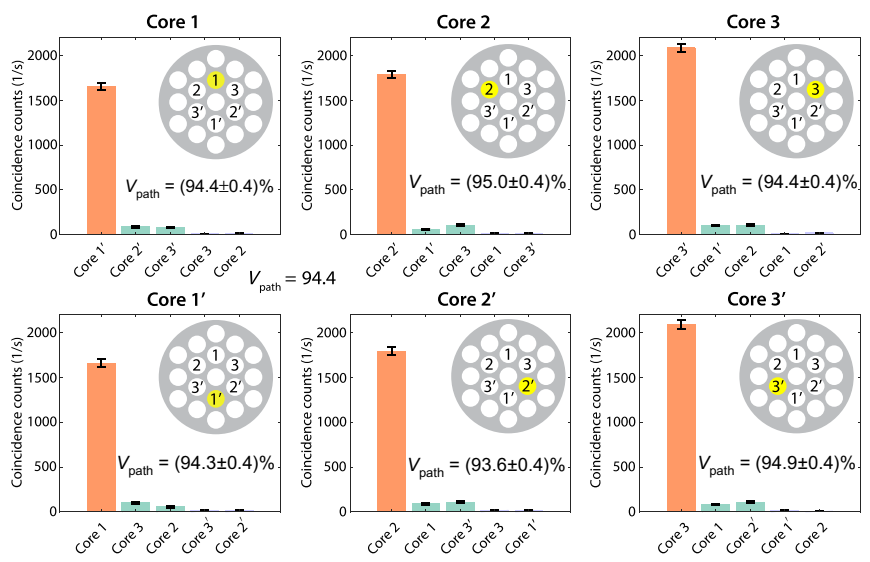

FIG. 2. Coincidence-based correlation measurements for the cores of the inner ring of the 19-core fiber. Each graph shows the coincidence counts measured between the core $m$ (highlighted in yellow) and all the other cores in the inner ring. The orange bar indicates the maximum coincidence counts observed between the opposite cores $m$ and $m^{\prime}$. The green bars are the coincidence counts between the cores $m$ and the neighbor cores of the core $m^{\prime}$. The last two bars in purple correspond to the coincidence counts between core $m$ and its neighbor cores. Pronounced correlations between opposite cores are reflected by the obtained values of the path visibilities indicated in each graph. The experimental error bars are calculated assuming Poissonian counting statistics. 

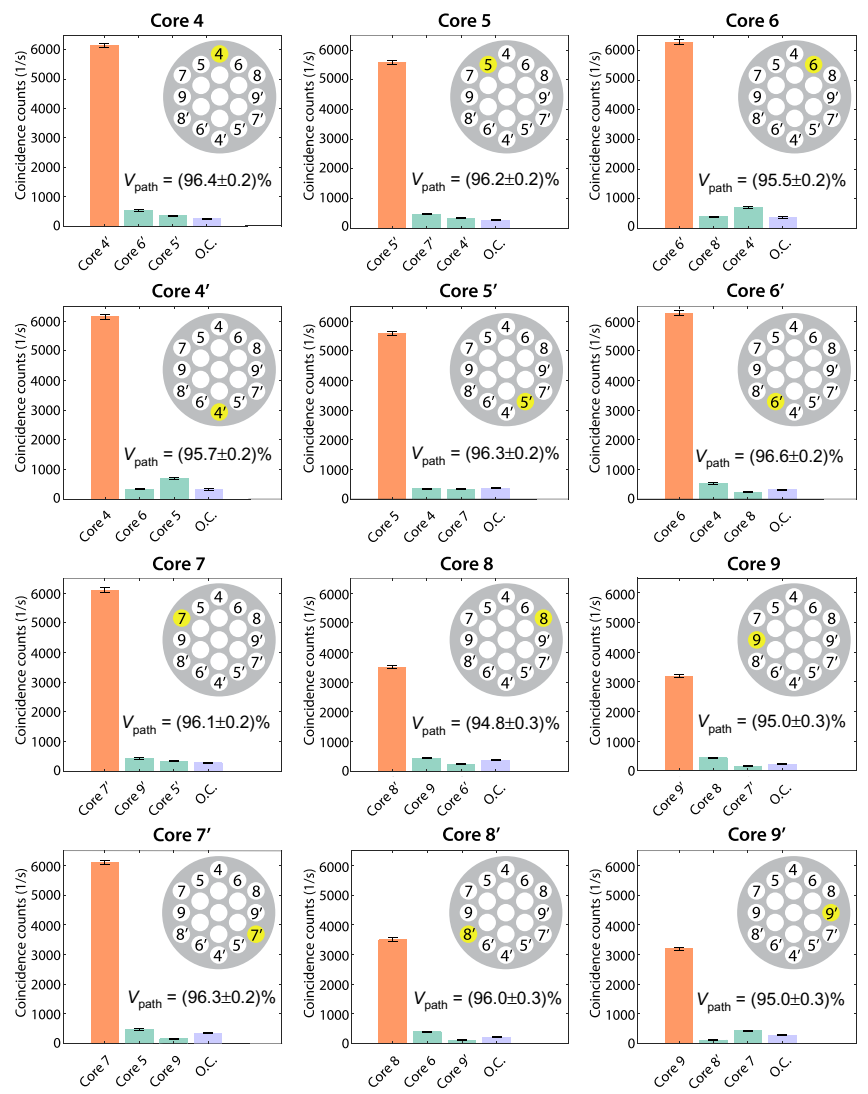

FIG. 3. Coincidence-based correlation measurements for the cores of the outer ring of the 19-core fiber. Each graph shows the coincidence counts measured between the core $m$ (highlighted in yellow) and all the other cores in the outer ring. The orange bar indicates the maximum coincidence counts observed between the opposite cores $m$ and $m^{\prime}$. The green bars are the coincidences between the cores $m$ and neighbor cores of the core $m^{\prime}$. The last purple bar is the sum of the other combination (O.C.) coincidence counts between the core $m$ and its other eight neighbor cores in the outer ring. This indicates strong correlations between opposite cores. The path visibility of each core is indicated in the graphs. The experimental error bars are calculated by assuming Poissonian counting statistics.

with the opposite core and low cross-correlations with the remaining cores.

To quantify these results, we introduce a measure that evaluates these correlations and the amount of crosstalk. In analogy to the polarization visibility [44], we introduce a corresponding measure, path visibility, which is defined by the coincidence counts between all possible combinations of cores within the considered ring:

$$
V_{\text {path }}(m)=\frac{C_{m m^{\prime}}-\left(\sum_{l \neq m, m^{\prime}} C_{m l}\right) / N}{C_{m m^{\prime}}+\left(\sum_{l \neq m, m^{\prime}} C_{m l}\right) / N} .
$$

Here $V_{\text {path }}(m)$ is the path visibility associated with the core $m, C_{m m^{\prime}}$ is the number of coincidences between the core $m$ and the opposite core $m^{\prime}, C_{m l}\left(l \neq m, m^{\prime}\right)$ is the number of coincidences between the core $m$ and the other cores in the same ring. Here the weighting of the sum by $1 / N, N$ being the number of nonopposite combinations, assures a balanced consideration of wanted (opposite) and unwanted (nonopposite) correlations. A high value of $V_{\text {path }}(m)$ close to 1 demonstrates strong correlations between opposite cores $\left(m\right.$ and $\left.m^{\prime}\right)$ and low crosstalk with the other cores.

The high path correlations in our experiment are reflected by the obtained values of the path visibilities. Our measurements show that the path visibility is greater than $(93.6 \pm 0.4) \%$ for all the inner cores $\left(1-3^{\prime}\right)$ and greater than $(94.8 \pm 0.3) \%$ for all the outer cores (4-9'), where we reach values up to $(96.6 \pm 0.2) \%$. We have thus confirmed that the photon pairs are coupled to and distributed over the MCF in a way that the spatial correlations in the SPDC emission are mapped to the cores (path). Importantly, this enables multiphoton experiments with up to 12 photons simultaneously using the outer ring of our MCF.

\section{POLARIZATION VISIBILITY}

Upon verifying that our setup features the reliable distribution of spatially correlated photon pairs through the MCF, we measure the quality of the polarization entangled state between opposite cores [see Eq. (A1)]. For this purpose, we carry out a series of polarization visibility [44] measurements on opposite channels in horizontal-vertical (HV) and diagonal-antidiagonal (DA) bases. For this measurement, polarization analyzing modules are connected to opposite cores (Fig. 6 in Appendix A, module b). The polarization modules consist of a half-wave plate (HWP) and a polarizing beam splitter (PBS) with a SNSPD connected to the transmitted and reflected output ports of the PBS. Through polarization compensation, two mutually unbiased bases are set and no active control or compensation is needed. For each pair of cores and analyzer setting, three pairs for the inner ring and six pairs for the outer ring, we accumulated the data for $60 \mathrm{~s}$.

To study the dependence on the polarization-measurement settings, one polarization module is set horizontal or diagonal, while the second module is scanned for the full range of HWP angles, from $0^{\circ}$ to $360^{\circ}$ in steps of $20^{\circ}$. The recorded coincidence data together with the corresponding fits are shown in Fig. 4 for the inner and outer rings. The expected oscillatory behavior for polarization entangled states is observed.

In order to certify the polarization entanglement, we measure the polarization visibility for each pair of opposite channels (see Appendix B for details). All the combinations exhibit visibilities greater than $(84.20 \pm 0.04) \%$ in the HV bases and $(83.60 \pm 0.04) \%$ in the DA bases. We reached up to $(95.15 \pm 0.04) \%$ in the best case. In all cases, the polarization visibilities are above $81 \%$, the threshold of quantum key distribution (QKD). Minor differences in visibilities are caused by the fact that the 
(a)
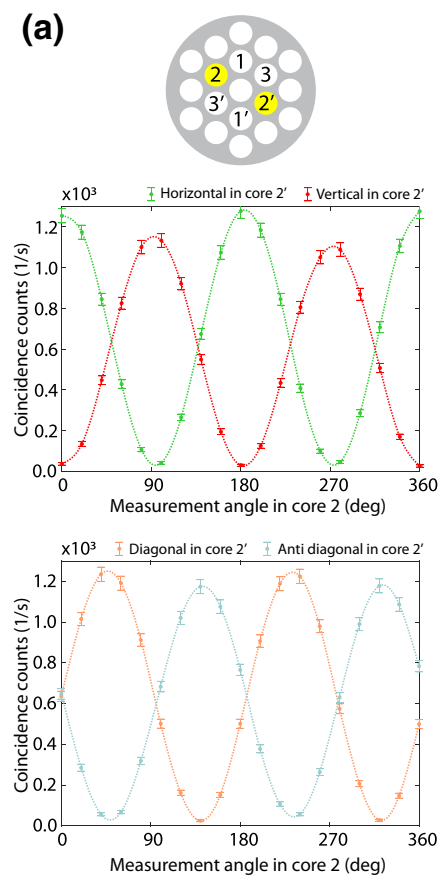

(b)
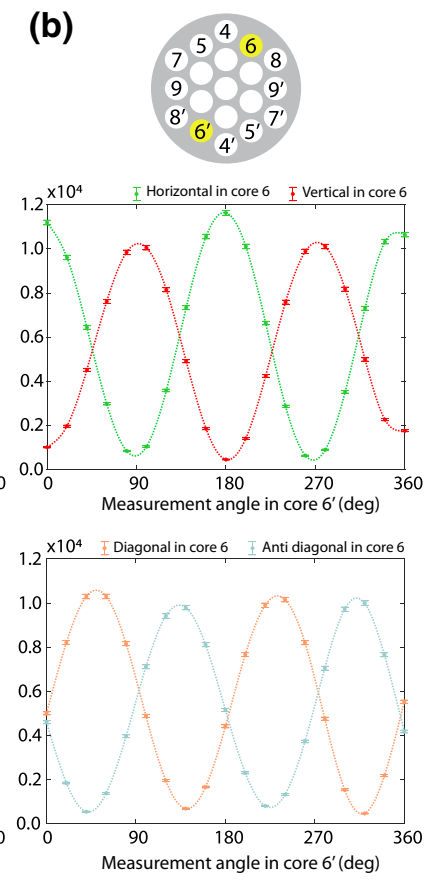

FIG. 4. Coincidence count rates of polarization-entangled photon pairs. For opposite cores, (a) 2-2' (inner ring) and (b) 6-6' (outer ring), the coincidence counts are recorded for four different measurement angles [horizontal (green), vertical (red), diagonal (orange), and antidiagonal (blue)] set in one polarization module (core 2' and core 6) while stepwise changing the angle in the second polarization module (core 2 and core 6 '). The error bars are calculated by assuming Poissonian counting statistics.

cores and the coupling into the cores of the MCF are not perfectly identical [42].

\section{FURTHER ANALYSIS AND APPLICATIONS}

In this section, we provide further analysis on the transferred entangled states and their applications. First, we calculate lower bounds on the quantum state fidelities with respect to a maximally entangled state. Second, we analyze the achievable secure key rate of our setup in a QKD implementation. Third, we perform a Bell-type experiment violating a CHSH inequality. Furthermore, we provide a long-term stability analysis of the setup.

\section{A. Quantum state fidelity}

In order to provide further analysis of the created and transmitted entangled states, we consider the quantum state fidelity with the maximally entangled target Bell state $\left|\Phi^{+}\right\rangle_{\text {pol }}$. From the measurements of nonclassical polarization-correlation visibilities in the HV and DA bases for each pair of opposite channels, we can calculate a lower bound on the Bell state fidelity given by $\mathcal{F}_{\text {lower }}=$ $\left(V_{\mathrm{HV}}+V_{\mathrm{DA}}\right) / 2$; see, e.g., Refs. [45,46]. This allows us to provide a (conservative) evaluation of the distributed entangled states. The minimal obtained value of the lower bound on the fidelity is $(83.90 \pm 0.04) \%$. In the inner ring the maximal value of $\mathcal{F}_{\text {lower }}$ is $(94.56 \pm 0.04) \%$ and in the outer ring we reach a value of $(94.64 \pm 0.02) \%$. These obtained lower bounds on the fidelity further underline the quality of the transferred polarization entanglement.

\section{B. Achievable secure key rates}

So far, we have focused on the quality of the distributed quantum correlations through the MCF. We now determine the achievable secure key rate when our setup is used in a QKD scenario. Here, it is important to stress that it is possible to create a key between each pair of opposite cores. Thus, it is possible to implement a multiplexed communication system, enabling a high-throughput entanglement-based QKD. The total secure key rate is therefore given by

$$
R_{\mathrm{tot}}^{s}=\sum_{m}^{N} R_{m}^{s},
$$

where $R_{m}^{s}$ is the secure key rate achievable between the $m$ th pair of cores and $N$ is the total number of opposite cores. We calculate each $R_{m}^{s}$ based on the recorded coincidences and polarization visibilities (determining the quantum bit errors); cf. Appendix D for details. From the inner and outer rings, we achieve an averaged key rate per pair of 2.3 and $5.7 \mathrm{kHz}$, respectively. By exploiting our SDM scheme, we therefore obtain total key rates $R_{\text {tot }}^{s}$ from the inner and outer rings of 7 and $34 \mathrm{kHz}$, respectively. The difference in the key rates of the inner and outer rings is due to the different crystal temperatures, which influence the SPDC emission cone and spatial correlation. Our results clearly show that the achievable key rate scales with the number of used pairs of cores, demonstrating the advantage and potential of quantum state multiplexed QKD through the MCF.

\section{Bell inequality violation}

We further measure the Bell-type CHSH inequality in each pair of channels (see Appendix $\mathrm{C}$ for more details). The polarization modules are prepared in the theoretical optimum values [47], whose angles are $\left(22.5^{\circ}\right.$, $\left.67.5^{\circ}\right)$ and $\left(0^{\circ}, 45^{\circ}\right)$. With the combination of the four bases in both modules, we measure the CHSH inequality, which is violated in both rings. In every pair of opposite cores, the $S$ parameters obtained are higher than $2.30 \pm 0.01$, which is evidence that the quantum features are preserved through the MCF. Moreover, we reach a maximum value of $2.73 \pm 0.03$, which corresponds to the pair of cores with the highest visibility, (93.96 \pm $0.04) \%$ in the HV bases and $(95.15 \pm 0.04) \%$ in the DA bases. 

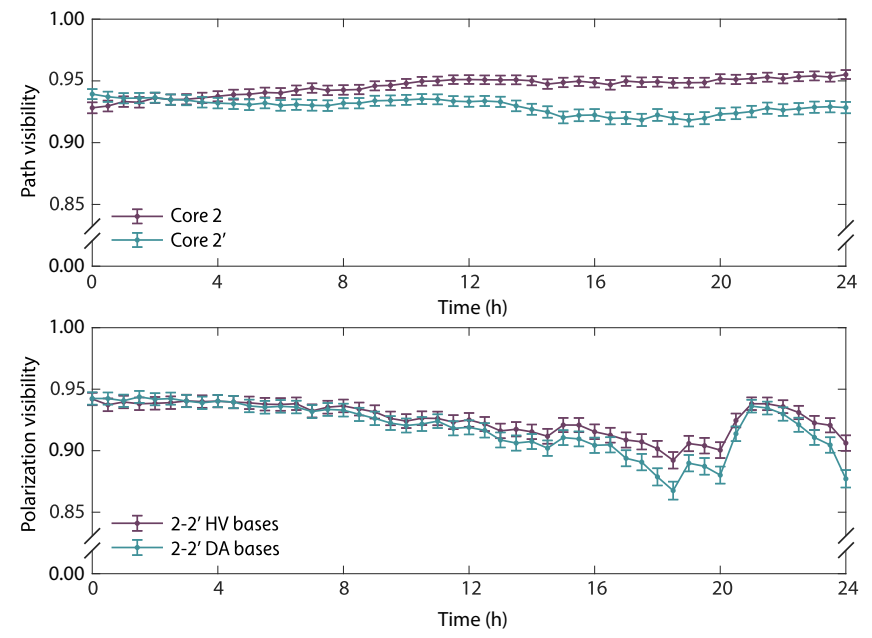

FIG. 5. Long-term measurements on the entangled photon state over a $411 \mathrm{~m}$ long MCF. A measurement for the path (upper plot) and polarization (lower plot) visibilities over a period of 24 $\mathrm{h}$ for one pair of inner ring cores. The visibilities are calculated from the measurement in each basis integrated over $60 \mathrm{~s}$, and the error bars are calculated by assuming Poissonian counting statistics for each point. The results demonstrate stable performance of our MCF implementation for the distribution of correlated photon pairs.

\section{Long-term stability}

In order to demonstrate the stability of our setup, we perform two long-term measurements of the path and polarization visibilities for one pair of cores over a period of 24 h. During this period, the coincidence counts are collected every $30 \mathrm{~min}$ for a period of $60 \mathrm{~s}$. For the polarization measurement, the visibilities in the HV and DA bases are measured alternately. As shown in the Fig. 5, both polarization and path visibilities are stable with only marginal fluctuations for at least $24 \mathrm{~h}$ without any external alignment or active stabilization. In the case of the path visibility, the minimum value is $(91.8 \pm 0.5) \%$ and the maximum $(95.5 \pm 0.4) \%$. The polarization visibility stayed in the range of values from $(86.8 \pm 0.7) \%$ to $(94.4 \pm 0.5) \%$. The residual changes in the laboratory environment do change the polarization state more severely than the path visibility.

\section{DISCUSSION}

We have successfully implemented the distribution of polarization entanglement multiplexed in the path degree of freedom through a 19-core MCF. We experimentally demonstrated the high quality with which the spatial modes are independently coupled into the MCF and quantified the resulting spatial correlations by high values of the introduced measure of path visibility of up to $(96.6 \pm$ $0.2) \%$. Furthermore, we certified polarization entanglement between each opposite channel through high polarization visibilities and violating the $\mathrm{CHSH}$ inequality.
That visibility was maintained over a spooled $411 \mathrm{~m}$ long MCF without any active polarization compensation. We illustrated the stability of our setup given by path and polarization visibility measurements over $24 \mathrm{~h}$, which is an important characteristic for future applications in real-world conditions outside the laboratory.

Exploiting the existing transverse momentum correlations in the emission of the SPDC source, we were able to collect polarization-entangled photon pairs into several pairs of opposite channels from a single SPDC source. Hence, our scheme is based on the parallel employment of quantum correlations in several degrees of freedom. The designed lens configuration allows us to access spatially separated rings and avoids crosstalk between neighboring cores by adapting the proper crystal temperature. Importantly, the achieved polarization visibilities allow the implementation of quantum information applications such as cryptographic protocols. Our scheme provides a substantial advantage in terms of resources and versatility. Because of the scalability and ease of upgrading without changing the entanglement source or the type of quantum state produced. Therefore, the presented experiment opens an efficient pathway to SDM in quantum information protocols.

An essential requirement for the multiplexed transfer of polarization-entangled photon pairs through the MCF is that the entangled photon pairs can be identified as a pair at the receiver. We realized this by mapping the momentum correlations of the photons to the cores of the MCF. Notably, this cannot be achieved by just randomly distributing the SPDC photons (without exploiting additional quantum correlations) through the MCF.

Our experiment can be efficiently adapted to other types of multicore fibers [27] and extended to using more cores at the same time by optimizing the source characteristic for strong momentum correlation, e.g., by changing the crystal length or pump waist. Likewise, the entanglement source can be adapted with different crystal configurations to generate photon pairs in more than one emission cone [48]. Additionally, the development of new MCFs with a higher number of cores allows for an increase in the number of independently distributed photon pairs [49]. At the same time, the phase-matching conditions remain a tunable parameter allowing for the reliable collection of photons into more channels.

Quantum SDM in optical fibers opens access to implementing QKD schemes for high-throughput quantum communications. Besides this, our implementation of the SDM with polarization-entangled photons can be combined with other degrees of freedom, e.g., the wavelength [50] or time bin [25], for enhancing communication capacity, and can be adapted for multiuser quantum network applications [22,51]. Furthermore, it provides a feasible way of implementing QKD based on position-momentum variables without time-consuming fiber scans [52] as all modes are 
guided simultaneously through the fiber. The next step is to ensure compatibility of the implemented SDM optical network with quantum technological applications, such as quantum communication systems.

Moreover, the presented experiment enables a variety of experimental applications: in combination with MCFintegrated multiport beam splitters [53], our experiment can be extended to a platform for quantum network applications. And the simultaneous coherent collection of several photons could be used for multiphoton interference experiments [54,55] or the implementation of multimode multipartner quantum metrology [56].

\section{ACKNOWLEDGMENTS}

We acknowledge funding by the European Union's Horizon 2020 programme Grant No. 857156 (OpenQKD) and the Austrian Academy of Sciences. J.C.A.-Z. and R.A.-C. acknowledge support from the US Army Research Office (W911NF1710553) and the National Science Foundation (ECCS-1711230). E.A.O. and J.F. acknowledge ANID for financial support [Becas de doctorado en el extranjero "Becas Chile"/2016 (Grant No. 72170402) and 2015 (Grant No. 72160487)].

\section{APPENDIX A: ENTANGLED PHOTON SOURCE}

In our work, we use a polarization-entangled source based on a type- 0 phase-matched SPDC process to distribute momentum correlation of photon pairs by a MCF.
The experimental setup is shown in Fig. 6. The entanglement photon pairs are generated in a $40 \mathrm{~mm}$ long magnesium-oxide-doped periodically poled lithium niobate $(\mathrm{MgO}: \mathrm{ppLN})$ crystal with a poling period of $\Lambda=$ $19.2 \mu \mathrm{m}$, pumped by a continuous-wave laser at a center wavelength of $775.07 \mathrm{~nm}$. The MgO:ppLN crystal is placed in a Sagnac interferometer without any additional active stabilization or compensation [57]. If the pump photons propagate clockwise (counterclockwise) in the Sagnac loop, the down-converted photons will be horizontally polarized $\left|H_{s}, H_{i}\right\rangle$ (vertically polarized $\left|V_{s}, V_{i}\right\rangle$ ). Under perfect alignment, while the crystal is pumped in both directions, the down-converted photons will be in a superposition of horizontal and vertical polarization:

$$
\left|\Phi^{+}\right\rangle=\frac{1}{\sqrt{2}}\left(\left|H_{s}, H_{i}\right\rangle+\left|V_{s}, V_{i}\right\rangle\right)
$$

The pump beam is spectrally cleaned with a long-pass filter $\left(\lambda_{\text {cut-off }}=780 \mathrm{~nm}\right)$. An interference filter $(\lambda=1550 \pm 3$ $\mathrm{nm})$ is used to select the degenerated down-converted photons.

The far-field plane of the crystal is formed by the lens $L_{1}$ with focal length $f_{1}=200 \mathrm{~mm}$. Then, the photon pairs in the far-field plane are coupled into the MCF cores by an imaging system consisting of the lenses $L_{2}\left(f_{2}=150\right.$ $\mathrm{mm})$ and $L_{3}\left(f_{3}=4.51 \mathrm{~mm}\right)$. This demagnified image of the down-converted photon pairs ensures that the emission

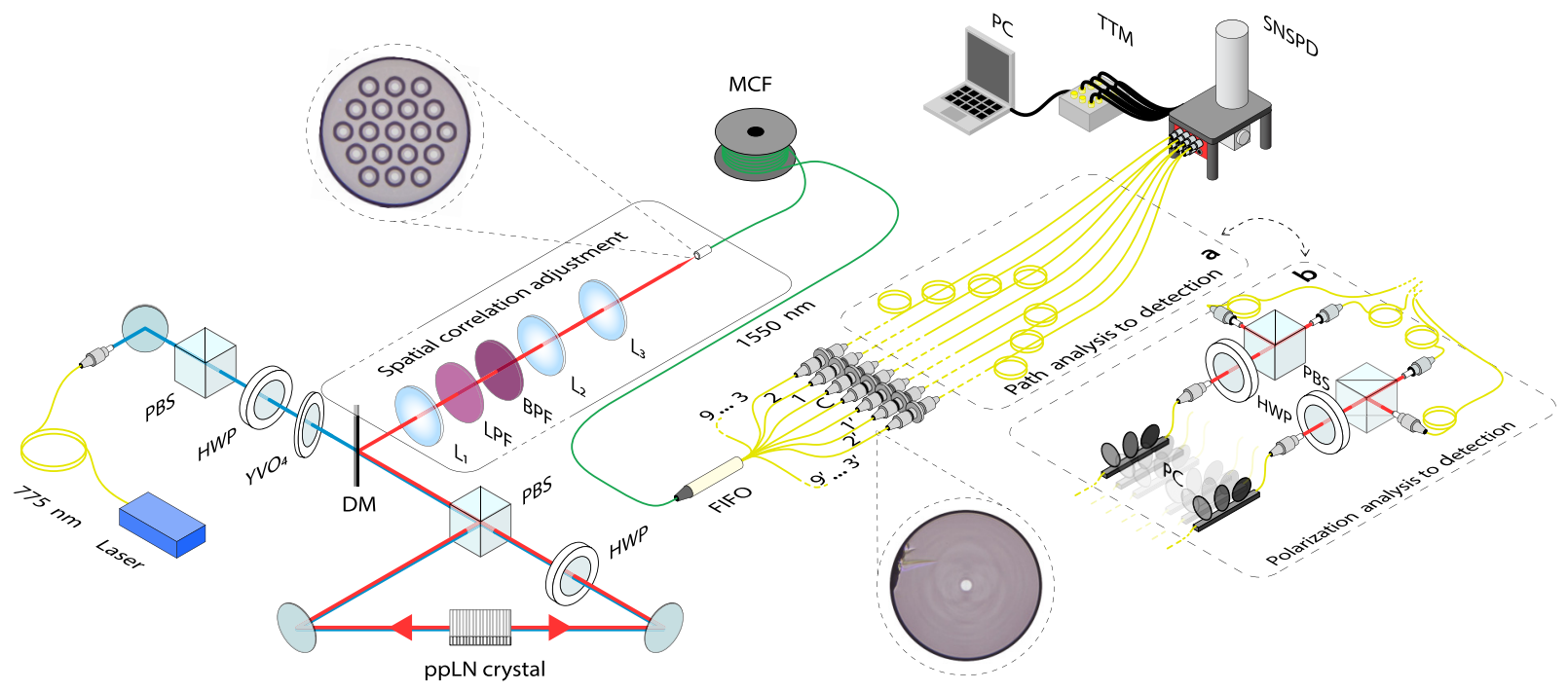

FIG. 6. Experimental setup. A MgO:ppLN crystal is pumped into a Sagnac configuration by a continuous-wave laser at 775.07 nm (blue line) to create polarization-entangled photon pairs with a center wavelength of $1550 \mathrm{~nm}$ (red line). The correlated photon pairs are distributed through opposite channels (cores) of the MCF with detection modules for the (a) path visibility measurement and (b) polarization visibility measurement. The 19 cores are split into individual SMF via a FIFO device. In both scenarios the photons are detected using a SNSPD with four channels. The efficiency of the detectors depends on the photon polarization, so a polarization controller is used to optimize the detection efficiency before each channel. $\mathrm{YVO}_{4}$ denotes the yttrium orthovanadate plate, DM denotes the dichroic mirror, LPF denotes the long-pass filter, BPF denotes the band-pass interference filter, and PC denotes the polarization controller. 
cone diameter covers all cores of a corresponding ring of cores.

By a scan of the crystal temperature, it can be verified that the emission cone, as seen in the Fourier plane, opens symmetrically around the center. If the maximum count rate is reached at the same temperature for all the cores with the same center-to-core distances, then the end face of the MCF is aligned with the emission cone. Afterwards, the optimal temperature for each ring is chosen, at which the amount of coincidence counts between opposite cores is maximal. In contrast, the amount of coincidences between nonopposite cores is low. For the inner and outer rings, the crystal temperature is set at $T_{1}=82.5^{\circ} \mathrm{C}$ and $T_{2}=82^{\circ} \mathrm{C}$, respectively. For that temperature, the inner (outer) ring is completely illuminated by the SPDC emission cone. Note how the outer ring has two core-to-core distances $(54.5 \mu \mathrm{m}$ and $64.5 \mu \mathrm{m}$ ), making a circular projection more difficult. Nevertheless, our implementation shows reliable coupling of photons with highly preserved correlations even in this configuration.

\section{APPENDIX B: ALIGNMENT AND CHARACTERIZATION OF THE POLARIZATION STATE}

In order to characterize the polarization correlations of the quantum state $\left|\Phi^{+}\right\rangle$between the spatial modes correlated in the far-field plane, we select two opposite cores and connect them to the polarization analyzing module. The polarization visibility is measured in two mutually unbiased bases to quantify the polarization entanglement. The polarization state of the photons is compensated in the HV and DA bases by fiber polarization controllers and by changing the polarization of the pump beam with a phase plate $\mathrm{YVO}_{4}$, respectively. Visibility in the HV basis (the expression for DA-basis is analogous) is given by

$$
V_{\mathrm{HV}}=\frac{C_{\mathrm{HH}}+C_{\mathrm{VV}}-C_{\mathrm{HV}}-C_{\mathrm{VH}}}{C_{\mathrm{HH}}+C_{\mathrm{VV}}+C_{\mathrm{HV}}+C_{\mathrm{VH}}}
$$

where $C_{S_{1}, s_{2}}$ is the number of coincidence counts for each polarization setting of the two opposite cores. The same procedure is repeated for each pair of opposite cores.

\section{APPENDIX C: CHSH MEASUREMENTS}

The CHSH inequality for two qubits states requires that $|S| \leq 2$, where the maximum value in quantum mechanics corresponds to the Tsirelson's bound $2 \sqrt{2}$ and the $S$ value is calculated by considering the coincidence counts of four combinations of settings in the polarization modules,

$$
S=E\left(a_{1}, b_{1}\right)+E\left(a_{2}, b_{1}\right)+E\left(a_{1}, b_{2}\right)-E\left(a_{2}, b_{2}\right)
$$

The correlation functions $E\left(a_{i}, b_{j}\right)$ are calculated from

$$
E\left(a_{i}, b_{j}\right)=\frac{C\left(a_{i}, b_{j}\right)+C\left(a_{i}^{\prime}, b_{j}^{\prime}\right)-C\left(a_{i}^{\prime}, b_{j}\right)-C\left(a_{i}, b_{j}^{\prime}\right)}{C\left(a_{i}, b_{j}\right)+C\left(a_{i}^{\prime}, b_{j}^{\prime}\right)+C\left(a_{i}^{\prime}, b_{j}\right)+C\left(a_{i}, b_{j}^{\prime}\right)},
$$

where $C\left(a_{i}, b_{j}\right)$ corresponds to the coincidence counts measured at angles $\left(a_{i}, b_{j}\right)$. We refer to the coincidence counts associated with the reflection output ports of the PBS as $a_{i}^{\prime}$ and $b_{j}^{\prime}$. We measure the $S$ value with polarization analyzers at angles $\left(0^{\circ}, 45^{\circ}\right)$ and $\left(22.5^{\circ}, 67.5^{\circ}\right)$.

\section{APPENDIX D: ESTIMATION OF THE KEY RATE}

The secret key rate for each pair of opposite cores given in the text has been estimated based on $[58,59]$

$$
\begin{aligned}
R_{m}^{s}= & \frac{1}{2} C_{\mathrm{HV}}\left[1-(1+f) H_{2}\left(\frac{1-V_{\mathrm{HV}}}{2}\right)\right] \\
& +\frac{1}{2} C_{\mathrm{DA}}\left[1-(1+f) H_{2}\left(\frac{1-V_{\mathrm{DA}}}{2}\right)\right],
\end{aligned}
$$

where $C_{\mathrm{HV}}$ and $C_{\mathrm{DA}}$ are the total numbers of coincidence counts in the HV and DA bases, $H_{2}$ is the binary Shannon entropy, and we used a bidirectional error correction of $f=1.1$.

[1] W. K. Wootters and W. H. Zurek, A single quantum cannot be cloned, Nature 299, 802 (1982).

[2] A. K. Ekert, Quantum Cryptography Based on Bell's Theorem, Phys. Rev. Lett. 67, 661 (1991).

[3] N. Gisin, G. Ribordy, W. Tittel, and H. Zbinden, Quantum cryptography, Rev. Mod. Phys. 74, 145 (2002).

[4] F. Xu, X. Ma, Q. Zhang, H.-K. Lo, and J.-W. Pan, Secure quantum key distribution with realistic devices, Rev. Mod. Phys. 92, 025002 (2020).

[5] R. Ursin, F. Tiefenbacher, T. Schmitt-Manderbach, H. Weier, T. Scheidl, M. Lindenthal, B. Blauensteiner, T. Jennewein, J. Perdigues, P. Trojek, B. Ömer, M. Fürst, M. Meyenburg, J. Rarity, Z. Sodnik, C. Barbieri, H. Weinfurter, and A. Zeilinger, Entanglement-based quantum communication over 144 km, Nat. Phys. 3, 481 (2007).

[6] J. Yin, J.-G. Ren, H. Lu, Y. Cao, H.-L. Yong, Y.-P. Wu, C. Liu, S.-K. Liao, F. Zhou, Y. Jiang, X.-D. Cai, P. Xu, G.-S. Pan, J.-J. Jia, Y.-M. Huang, H. Yin, J.-Y. Wang, Y.-A. Chen, C.-Z. Peng, and J.-W. Pan, Quantum teleportation and entanglement distribution over 100-kilometre free-space channels, Nature 488, 185 (2012).

[7] S.-K. Liao et al., Satellite-Relayed Intercontinental Quantum Network, Phys. Rev. Lett. 120, 030501 (2018).

[8] B. Korzh, C. C. W. Lim, R. Houlmann, N. Gisin, M. J. Li, D. Nolan, B. Sanguinetti, R. Thew, and H. Zbinden, Provably secure and practical quantum key distribution over 307 $\mathrm{km}$ of optical fibre, Nat. Photonics 9, 163 (2015).

[9] A. Boaron, G. Boso, D. Rusca, C. Vulliez, C. Autebert, M. Caloz, M. Perrenoud, G. Gras, F. Bussières, M.-J. Li, D. Nolan, A. Martin, and H. Zbinden, Secure Quantum key 
Distribution Over 421 km of Optical Fiber, Phys. Rev. Lett. 121, 190502 (2018).

[10] S. Wengerowsky, S. K. Joshi, F. Steinlechner, J. R. Zichi, B. Liu, T. Scheidl, S. M. Dobrovolskiy, R. van der Molen, J. W. N. Los, V. Zwiller, M. A. M. Versteegh, A. Mura, D. Calonico, M. Inguscio, A. Zeilinger, A. Xuereb, and R. Ursin, Passively stable distribution of polarisation entanglement over $192 \mathrm{~km}$ of deployed optical fibre, Npj Quantum Inf. 6, 1 (2020).

[11] L.-J. Wang, L.-K. Chen, L. Ju, M.-L. Xu, Y. Zhao, K. Chen, Z.-B. Chen, T.-Y. Chen, and J.-W. Pan, Experimental multiplexing of quantum key distribution with classical optical communication, Appl. Phys. Lett. 106, 081108 (2015).

[12] D. J. Richardson, J. M. Fini, and L. E. Nelson, Spacedivision multiplexing in optical fibres, Nat. Photonics 7 , 354 (2013).

[13] P. Sillard, M. Bigot-Astruc, and D. Molin, Few-mode fibers for mode-division-multiplexed systems, J. Lightwave Technol. 32, 2824 (2014).

[14] J. van Weerdenburg, R. Ryf, J. C. Alvarado-Zacarias, R. A. Alvarez-Aguirre, N. K. Fontaine, H. Chen, R. Amezcua-Correa, Y. Sun, L. Grüner-Nielsen, R. V. Jensen, R. Lingle, T. Koonen, and C. Okonkwo, 138-tb/s modeand wavelength-multiplexed transmission over six-mode graded-index fiber, J. Lightwave Technol. 36, 1369 (2018).

[15] P. Sillard, D. Molin, M. Bigot-Astruc, A. Amezcua-Correa, $\mathrm{K}$. de Jongh, and F. Achten, $50 \mu \mathrm{m}$ multimode fibers for mode division multiplexing, J. Lightwave Technol. 34, 1672 (2016).

[16] R. G. H. van Uden, R. A. Correa, E. A. Lopez, F. M. Huijskens, C. Xia, G. Li, A. Schülzgen, H. de Waardt, A. M. J. Koonen, and C. M. Okonkwo, Ultra-high-density spatial division multiplexing with a few-mode multicore fibre, Nat. Photonics 8, 865 (2014).

[17] R. Ryf, J. C. Alvarado, B. Huang, J. Antonio-Lopez, S. H. Chang, N. K. Fontaine, H. Chen, R. Essiambre, E. Burrows, R. Amezcua-Correa, T. Hayashi, Y. Tamura, T. Hasegawa, and T. Taru, in ECOC 2016 - Post Deadline Paper; 42nd European Conference on Optical Communication (2016), p. 1.

[18] K. Saitoh and S. Matsuo, Multicore fiber technology, J. Lightwave Technol. 34, 55 (2016).

[19] A. M. Velazquez-Benitez, J. C. Alvarado, G. LopezGalmiche, J. E. Antonio-Lopez, J. Hernández-Cordero, J. Sanchez-Mondragon, P. Sillard, C. M. Okonkwo, and R. Amezcua-Correa, Six mode selective fiber optic spatial multiplexer, Opt. Lett. 40, 1663 (2015).

[20] J. C. Alvarado-Zacarias, N. K. Fontaine, J. E. AntonioLopez, Z. S. Eznaveh, M. S. Habib, H. Chen, R. Ryf, D. Van Ras, P. Sillard, C. Gonnet, A. Amezcua-Correa, S. G. Leon-Saval, and R. A. Correa, in 2018 Optical Fiber Communications Conference and Exposition (OFC) (2018), p. 1.

[21] J. Chen, G. Wu, L. Xu, X. Gu, E. Wu, and H. Zeng, Stable quantum key distribution with active polarization control based on time-division multiplexing, New J. Phys. 11, 065004 (2009).

[22] S. Wengerowsky, S. K. Joshi, F. Steinlechner, H. Hübel, and R. Ursin, An entanglement-based wavelength-multiplexed quantum communication network, Nature 564, 225 (2018).
[23] Z.-Y. Chen, L.-S. Yan, Y. Pan, L. Jiang, A.-L. Yi, W. Pan, and B. Luo, Use of polarization freedom beyond polarization-division multiplexing to support high-speed and spectral-efficient data transmission, Light: Sci. Appl. 6, e16207 (2016).

[24] S. Leedumrongwatthanakun, L. Innocenti, H. Defienne, T. Juffmann, A. Ferraro, M. Paternostro, and S. Gigan, Programmable linear quantum networks with a multimode fibre, Nat. Photonics 14, 139 (2019).

[25] L. Cui, J. Su, X. Li, and Z. Y. Ou, Distribution of entangled photon pairs over few-mode fibers, Sci. Rep. 7, 1 (2017).

[26] W. Löffler, T. G. Euser, E. R. Eliel, M. Scharrer, P. S. J. Russell, and J. P. Woerdman, Fiber Transport of Spatially Entangled Photons, Phys. Rev. Lett. 106, 240505 (2011).

[27] M. Tang, in Handbook of Optical Fibers (Springer Singapore, 2019), p. 895.

[28] G. B. Xavier and G. Lima, Quantum information processing with space-division multiplexing optical fibres, Commun. Phys. 3, 1 (2020).

[29] J. F. Dynes, S. J. Kindness, S. W.-B. Tam, A. Plews, A. W. Sharpe, M. Lucamarini, B. Fröhlich, Z. L. Yuan, R. V. Penty, and A. J. Shields, Quantum key distribution over multicore fiber, Opt. Express 24, 8081 (2016).

[30] D. Bacco, B. D. Lio, D. Cozzolino, F. D. Ros, X. Guo, Y. Ding, Y. Sasaki, K. Aikawa, S. Miki, H. Terai, T. Yamashita, J. S. Neergaard-Nielsen, M. Galili, K. Rottwitt, U. L. Andersen, T. Morioka, and L. K. Oxenløwe, Boosting the secret key rate in a shared quantum and classical fibre communication system, Commun. Phys. 2, 1 (2019).

[31] G. Cañas, N. Vera, J. Cariñe, P. González, J. Cardenas, P. W. R. Connolly, A. Przysiezna, E. S. Gómez, M. Figueroa, G. Vallone, P. Villoresi, T. F. da Silva, G. B. Xavier, and G. Lima, High-dimensional decoy-state quantum key distribution over multicore telecommunication fibers, Phys. Rev. A 96, 022317 (2017).

[32] Y. Ding, D. Bacco, K. Dalgaard, X. Cai, X. Zhou, K. Rottwitt, and L. K. Oxenløwe, High-dimensional quantum key distribution based on multicore fiber using silicon photonic integrated circuits, Npj Quantum Inf. 3, 1 (2017).

[33] B. D. Lio, D. Cozzolino, N. Biagi, Y. Ding, K. Rottwitt, A. Zavatta, D. Bacco, and L. K. Oxenløwe, Path-encoded high-dimensional quantum communication over a $2-\mathrm{km}$ multicore fiber, Npj Quantum Inf. 7, 1 (2021).

[34] X.-M. Hu, W.-B. Xing, B.-H. Liu, D.-Y. He, H. Cao, Y. Guo, C. Zhang, H. Zhang, Y.-F. Huang, C.-F. Li, and G.-C. Guo, Efficient distribution of high-dimensional entanglement through $11 \mathrm{~km}$ fiber, Optica 7, 738 (2020).

[35] H. J. Lee, S.-K. Choi, and H. S. Park, Experimental demonstration of four-dimensional photonic spatial entanglement between multi-core optical fibres, Sci. Rep. 7, 1 (2017).

[36] H. J. Lee and H. S. Park, Generation and measurement of arbitrary four-dimensional spatial entanglement between photons in multicore fibers, Photon. Res. 7, 19 (2019).

[37] E. Gómez, S. Gómez, I. Machuca, A. Cabello, S. Pádua, S. Walborn, and G. Lima, Multidimensional Entanglement 
Generation with Multicore Optical Fibers, Phys. Rev. Appl. 15, 034024 (2021).

[38] M. Barbieri, G. Vallone, P. Mataloni, and F. De Martini, Complete and deterministic discrimination of polarization bell states assisted by momentum entanglement, Phys. Rev. A 75, 042317 (2007).

[39] R. Ceccarelli, G. Vallone, F. De Martini, P. Mataloni, and A. Cabello, Experimental Entanglement and Nonlocality of a Two-Photon Six-Qubit Cluster State, Phys. Rev. Lett. 103, 160401 (2009).

[40] H. J. Lee, H. Kim, M. Cha, and H. S. Moon, Generation of bright visible photon pairs using a periodically poled stoichiometric lithium tantalate crystal, Opt. Express 23, 14203 (2015).

[41] E. A. Ortega et al., (to be published).

[42] J. C. Alvarado-Zacarias, J. E. Antonio-Lopez, M. S. Habib, S. Gausmann, N. Wang, D. Cruz-Delgado, G. Li, A. Schülzgen, A. Amezcua-Correa, L.-A. Demontmorillon, P. Sillard, and R. Amezcua-Correa, in Optical Fiber Communication Conference (OFC) 2019 (Optical Society of America, 2019), p. Th3D.2.

[43] S. Walborn, C. Monken, S. Pádua, and P. Souto Ribeiro, Spatial correlations in parametric down-conversion, Phys. Rep. 495, 87 (2010).

[44] E. Wolf et al., Introduction to the Theory of Coherence and Polarization of Light (Cambridge University Press, Cambridge, U.K., 2007).

[45] B. B. Blinov, D. L. Moehring, L.-M. Duan, and C. Monroe, Observation of entanglement between a single trapped atom and a single photon, Nature 428, 153 (2004).

[46] X.-Y. Chang, D.-L. Deng, X.-X. Yuan, P.-Y. Hou, Y.Y. Huang, and L.-M. Duan, Experimental realization of an entanglement access network and secure multi-party computation, Sci. Rep. 6, 1 (2016).

[47] J. F. Clauser, M. A. Horne, A. Shimony, and R. A. Holt, Proposed Experiment to Test Local Hidden-Variable Theories, Phys. Rev. Lett. 23, 880 (1969).

[48] G. Vallone, R. Ceccarelli, F. De Martini, and P. Mataloni, Hyperentanglement of two photons in three degrees of freedom, Phys. Rev. A 79, 030301 (2009).

[49] Y. Sasaki, K. Takenaga, K. Aikawa, Y. Miyamoto, and T. Morioka, in 2017 Optical Fiber Communications Conference and Exhibition (OFC) (2017), p. 1.
[50] J. Pseiner, L. Achatz, L. Bulla, M. Bohmann, and R. Ursin, Experimental wavelength-multiplexed entanglement-based quantum cryptography, Quantum Sci. Technol. 6, 035013 (2021).

[51] S. K. Joshi, D. Aktas, S. Wengerowsky, M. Lončarić, S. P. Neumann, B. Liu, T. Scheidl, G. C. Lorenzo, Ž. Samec, L. Kling, A. Qiu, M. Razavi, M. Stipčević, J. G. Rarity, and R. Ursin, A trusted node-free eight-user metropolitan quantum communication network, Sci. Adv. 6, eaba0959 (2020).

[52] M. P. Almeida, S. P. Walborn, and P. H. Souto Ribeiro, Experimental investigation of quantum key distribution with position and momentum of photon pairs, Phys. Rev. A 72, 022313 (2005).

[53] J. C. ne, G. C. nas, P. Skrzypczyk, I. Šupić, N. Guerrero, T. Garcia, L. Pereira, M. A. S. Prosser, G. B. Xavier, A. Delgado, S. P. Walborn, D. Cavalcanti, and G. Lima, Multicore fiber integrated multi-port beam splitters for quantum information processing, Optica 7, 542 (2020).

[54] S. Agne, T. Kauten, J. Jin, E. Meyer-Scott, J. Z. Salvail, D. R. Hamel, K. J. Resch, G. Weihs, and T. Jennewein, Observation of Genuine Three-Photon Interference, Phys. Rev. Lett. 118, 153602 (2017).

[55] A. J. Menssen, A. E. Jones, B. J. Metcalf, M. C. Tichy, S. Barz, W. S. Kolthammer, and I. A. Walmsley, Distinguishability and Many-Particle Interference, Phys. Rev. Lett. 118, 153603 (2017).

[56] F. Albarelli, M. Barbieri, M. Genoni, and I. Gianani, A perspective on multiparameter quantum metrology: From theoretical tools to applications in quantum imaging, Phys. Lett. A 384, 126311 (2020).

[57] T. Kim, M. Fiorentino, and F. N. C. Wong, Phase-stable source of polarization-entangled photons using a polarization sagnac interferometer, Phys. Rev. A 73, 012316 (2006).

[58] X. Ma, C.-H. F. Fung, and H.-K. Lo, Quantum key distribution with entangled photon sources, Phys. Rev. A 76, 012307 (2007).

[59] S. P. Neumann, T. Scheidl, M. Selimovic, M. Pivoluska, B. Liu, M. Bohmann, and R. Ursin, Model for optimizing quantum key distribution with continuous-wave pumped entangled-photon sources, Phys. Rev. A 104, 022406 (2021). 